\title{
Embarazo adolescente en un Hospital de Paraguay durante el 2011
}

\author{
Beatriz Mercedes Cabañas Acosta ${ }^{1,2}$, Jorge Sebastián Escobar Salinas ${ }^{1,2}$
}

\section{RESUMEN}

Objetivo: Describir las características epidemiológicas del embarazo adolescente en el Hospital Regional de Coronel Oviedo, Paraguay.

Material y Métodos: Estudio observacional descriptivo de corte transversal, con muestreo no probabilístico de casos consecutivos, con revisión de historias clínicas de las embarazadas adolescentes que acudieron al Hospital Regional de Coronel Oviedo, Paraguay entre los meses enero y diciembre del año 2011.

Resultados: Se incluyeron 167 historias clínicas de mujeres embarazadas adolescentes, las cuales representaron el 40,7\% de todos los partos asistidos durante el año 2011. La franja etaria estuvo comprendida entre 13 y 19 años, con un promedio de edad de $17,47 \pm 1,42$. El $64,1 \%$ fueron de procedencia urbana. El $86,8 \%$ no usaba anticonceptivos y el $50,9 \%$ tenían una unión libre. Las complicaciones observadas fueron: ruptura prematura de membrana en el 10,2\%, infección de las vías urinarias en el $8,4 \%$ y óbito fetal en el $0,6 \%$ de los casos.

Conclusión: La prevalencia de embarazo adolescente en este estudio fue de 40,7\%; la mayoría de las mujeres no usaba ningún método anticonceptivo. La complicación más frecuente en este tipo de embarazo fue la ruptura prematura de membrana.

Palabras clave: embarazo adolescente, complicaciones del embarazo.

\section{Teen pregnancy in a Hospital in Paraguay in 2011}

\section{ABSTRACT}

Objective: To describe the epidemiological characteristics of teenage pregnancy in the Regional Hospital of Coronel Oviedo, Paraguay.

Material and Methods: Descriptive, observational cross-sectional study with consecutive non-probability sampling of cases, review of medical records of pregnant teenagers who attended the Regional Hospital of Coronel Oviedo, Paraguay between january and december 2011.

Results: 167 medical records of pregnant adolescents were included, which accounted for $40.7 \%$ of all births attended in 2011. The age range was between 13 and 19 years, with an average age of $17.47 \pm 1.42 .64 .1 \%$ were of urban origin. 86.8\% did not use contraception and $50.9 \% \mathrm{had}$ a free union. The observed complications were: premature rupture of membrane at $10.2 \%$, urinary tract infection at $8.4 \%$ and fetal death in $0.6 \%$ of cases.

Conclusion: The prevalence of teenage pregnancy in this study was $40.7 \%$; most women did not use any contraceptive method. The most common complication in this type of pregnancy was premature rupture of membrane.

Key words: teen pregnancy, pregnancy complications.

1. Estudiante de medicina. Facultad de Ciencias Médicas. Universidad Nacional de Caaguazú. Coronel Oviedo, Paraguay.

2. Sociedad científica de estudiantes de medicina - Universidad Nacional de Caaguazú. Coronel Oviedo, Paraguay. 


\section{INTRODUCCIÓN}

La Organización Mundial de la Salud (OMS) define a la adolescencia como el periodo de vida en el cual el individuo adquiere la capacidad reproductiva, además de pasar por una transformación física y psicológica, y establece que la adolescencia se divide en temprana (de los 10 a los 14 años) y tardía (de los 15 a los 19 años) (1).

En países desarrollados y en vía de desarrollo, el embarazo en la adolescencia tiene un gran impacto en la salud pública por las consecuencias que acarrean, tales como aumento de aborto provocado, inicio tardío del control pre natal y mal control pre natal por el inicio tardío. Algunas cifras indican que tres de cada cuatro embarazos en adolescentes no son deseados, y el cuarto de estos terminan en aborto (2).

En América Latina y el Caribe, la población adolescente representa el 19\% (3) y la prevalencia de embarazo en la adolescencia varía en cada país, dependiendo del nivel socioeconómico de la población de estudio; $20 \%$ en México (4), 20,33\% en Brasil (5), 39\% en Colombia (6), 12,2\% en Perú, 24,6\% en Nicaragua, $21,4 \%$ en Honduras y $15,6 \%$ en Bolivia (7).

Algunas características que encontramos en las madres adolescentes son: pareja inestable, deserción escolar (24,6\% según Osorio y Cols (8), rechazo de la familiar, bajo nivel socioeconómico; todo esto sumado a los factores físicos y biológicos como la inmadurez física y emocional se asocian a un mayor riesgo de mortalidad materno infantil (3).

Las complicaciones somáticas más frecuente en las madres adolescentes son: Anemia, Infección del tracto urinario, retardo en el crecimiento intrauterino, parto prematuro, bajo peso al nacer y complicaciones en el parto como hemorragias $\mathrm{y} / \mathrm{o}$ desgarro uterino $(9,10)$.

Debido a que el embarazo en la adolescencia sigue siendo a nivel mundial un impedimento para mejorar las condiciones educativas, económicas y sociales de las mujeres, la OMS recomienda implementar intervenciones combinadas como la promoción educativa y anticoncepción en las escuelas, comunidad y/o centros de atención en salud para disminuir los embarazos no deseados en adolescentes $(11,12)$.
Teniendo en cuenta la prevalencia en varios países subdesarrollado como el Paraguay resulta importante la determinación de esta problemática, si es que fuera el caso, como factores de riesgo para ciertas patologías o complicaciones relacionadas al embarazo.

El propósito de este estudio fue determinar la prevalencia de embarazo en la adolescencia en el Hospital Regional de Coronel Oviedo, Paraguay durante el 2011 e identificar las características epidemiológicas del embarazo adolescente y sus complicaciones.

\section{MATERIAL Y MÉTODOS}

Se realizó un estudio observacional descriptivo de corte transversal, con muestreo no probabilístico de casos consecutivos, durante los meses enero a diciembre del año 2011 en el Hospital Regional de Coronel Oviedo - Paraguay.

La población del estudio estuvo comprendida por mujeres embarazadas que ingresaron por el servicio de consultorio para su control prenatal en el Hospital Regional “Dr. Ángel Samudio" de Coronel Oviedo.

La Ciudad de Coronel Oviedo, Departamento de Caaguazú se encuentra ubicado en la región oriental del territorio Paraguayo, $25^{\circ} 25^{\prime} 00^{\prime \prime} \mathrm{S} 56^{\circ} 27^{\prime} 00^{\prime \prime} \mathrm{O}$; y según el último estudio de la Dirección de Planificaciones del Municipio cuenta con 120.632 hab. El estudio se realizó en el Hospital Regional “Dr. Ángel Samudio" de Coronel Oviedo. Dicho centro hospitalario pertenece a la $V$ región sanitaria del Ministerio de Salud Pública y Bienestar Social del Paraguay, categorizado como nivel de atención III, básico - complementario.

Se realizó una revisión y análisis de fuentes secundaria, basadas en los registros de 410 historias clínicas de mujeres embarazadas con edades comprendidas entre 13 y 45 años quienes acudían a su control de embarazo y posteriormente para el parto al Hospital Regional Coronel Oviedo en el periodo enero a diciembre del año 2011.

Fueron excluidas del estudio aquellas historias clínicas que pertenecían a mujeres embarazadas no adolescentes.

Se definió como mujer adolescentes aquellas cuya edad estaba comprendida entre 10 y 19 años (1). Las mujeres embarazadas adolescentes en el estudio 
fueron distribuidas en dos grupos; primer grupo con edades comprendidas de 10 a 15 años y el segundo grupo con edades comprendidas de 16 a 19 años. Se tuvo en cuenta el estado civil de la embarazada en el momento de la consulta y fue clasificado en unión libre o en concubinato, casada y soltera. La procedencia de la embarazada fue clasificada en zonas: urbana y rural, de acuerdo al lugar de residencia de la misma. El tipo de anticonceptivo utilizado en el momento del embarazo y si éste fue planeado o no. También fueron observados si presentaba infección urinaria. Las complicaciones registradas en el momento del parto fueron ruptura prematura de membrana y óbito fetal.

Antes de la revisión de las historias clínicas perinatales, se solicitó autorización para acceder a los datos del departamento de estadística del Hospital Regional de Coronel Oviedo, la solicitud fue aceptada por el Director del nosocomio, posterior a esto se procedió a la revisión de las historias clínicas y la elaboración de la base de datos.

Los datos proporcionados en las fichas perinatales fueron cargados inicialmente en una planilla Excel, luego del control de calidad de los datos y las variables, fueron exportados y analizados con el Programa Epidat para Windows versión 3.1, se realizó un análisis de cada variable y se presentan tablas de la frecuencia y prevalencia.
Toda la información de las historias clínicas utilizadas fue confidencial. Por parte de los investigadores no se divulgó, repitió o comentó la información recolectada de las historias clínicas.

\section{RESULTADOS}

De las 410 historias clínicas de embarazadas registradas durante el año 2011 en el Hospital Regional de Coronel Oviedo que acudieron al mencionado nosocomio para el control prenatal y posteriormente para el parto, fueron excluidas 243 historias clínicas pertenecientes a mujeres embarazadas no adolescentes. Del total de embarazadas, se incluyeron finalmente en el estudio 167 (40,7\%) de los registros pertenecientes a mujeres embarazas adolescentes.

La franja etaria de las embarazadas adolescentes estuvo comprendida entre 13 y 19 años $(17,47 \pm 1,42)$, $88,6 \%$ tenían entre 16 y 19 años. Del total de embarazadas adolescentes el $50,9 \%$ tenía en el momento del parto una unión libre con su pareja, el $64,1 \%$ procedía de la zona urbana, el $86,8 \%$ no usaba ningún método anticonceptivo, entre las que sí lo utilizaban el tipo hormonal fue el más frecuente y el $81,4 \%$ no planeó su embarazo (Tabla 1 ).

Tabla 1. Característica de adolescentes embarazadas en el Hospital Regional de Coronel Oviedo, enero a diciembre 2011

\begin{tabular}{|l|r|r|}
\hline \multicolumn{3}{|c|}{ n } \\
\hline Edad & 19 & $11,4 \%$ \\
\hline $10-15$ & 148 & $88,6 \%$ \\
\hline $16-19$ & 107 & $64,1 \%$ \\
\hline Procedencia & 60 & $35,9 \%$ \\
\hline Zona urbana & 85 & $50,9 \%$ \\
\hline Zona rural & 67 & $40,1 \%$ \\
\hline Estado civil & 15 & $9 \%$ \\
\hline Unión libre & 145 & $86,8 \%$ \\
\hline Solteras & 18 & $10,8 \%$ \\
\hline Casadas & 2 & $1,2 \%$ \\
\hline Tipo de anticonceptivo & 2 & $1,2 \%$ \\
\hline Ninguno & \multicolumn{2}{|l|}{} \\
\hline Hormonal & 31 & $18,6 \%$ \\
\hline Barrera & 136 & $81,4 \%$ \\
\hline Natural &
\end{tabular}


Las complicaciones halladas en las embarazadas adolescentes durante el embarazo fueron ruptura prematura de membrana en el 10,2\%, infección en las vías urinaria en el 8,4\% y óbito fetal en el 0,6\% (Tabla 2).

Tabla 2. Distribución de las variables clínicas de las embarazadas adolescentes en el Hospital Regional de Coronel Oviedo, enero a diciembre 2011

\begin{tabular}{|lcc|}
\hline & $\mathrm{n}$ & $\%$ \\
\hline Ruptura prematura de membrana & 17 & $10,2 \%$ \\
\hline Infección urinaria & 14 & $8,4 \%$ \\
\hline Óbito fetal & 1 & $0,6 \%$ \\
\hline
\end{tabular}

\section{DISCUSIÓN}

Los resultados obtenidos en la investigación realizada coinciden parcialmente con otras investigaciones similares, que apuntan que los partos en adolescentes como porcentaje de todos los partos oscilan entre alrededor del $2 \%$ en China y el $19 \%$ en América Latina y el Caribe $(1,3)$. La prevalencia de embarazo adolescente en este estudio fue del $40,7 \%$ lo cual resulta muy elevada en comparación al estudio realizado en el 2008 en la Ciudad de Asunción, Paraguay por el Centro Paraguayo de Estudio de Población, donde la prevalencia fue del 11,6\% (15). Como en otras casuísticas (13-17) el grupo etario predominante fue el de 15 a 19 años.

La mayoría de las embarazadas provenían de la zona urbana, al igual que en el estudio publicado por Padilla de Gil. (16) sin embargo, no concuerda con los datos nacionales obtenidos de las publicaciones del CEPEP, que concluye que el mayor porcentaje de adolescentes embarazadas provienen de la zona rural (15).

La mayor parte de los nacimientos de madres menores de 20 años se da dentro de una relación que se considera de cierta estabilidad o duración (15). Como se muestra en nuestros resultados, el menor porcentaje de madres adolescentes era soltera en el momento del parto.

El $81,4 \%$ de los embarazos observado en nuestro estudio, no fueron planeados. Esto demuestra la inefectividad de las campañas de prevención para disminuir la prevalencia de embarazo en los adolescentes. A pesar de la mayor disponibilidad de información y el fácil acceso a métodos anticonceptivo, la tasa de embarazo en los adolescentes va en aumento (16).
En el trabajo se observa cómo el $86,8 \%$ no usaba ningún método anticonceptivo, resultado similar se obtuvo en otras investigaciones en el Salvador (16). El anticonceptivo más frecuente fue del tipo hormonal hecho que concuerda con los resultados obtenidos por CEPEP (15), esto también revela que este tipo de anticonceptivo sigue siendo el más utilizado por las adolescentes.

Entre las complicaciones más frecuentes encontramos la ruptura prematura de membrana con un $10,2 \%$, complicación que también fue observada en un estudio con igual población (17). Otros estudios hallaron mayor prevalencia de infecciones urinarias en adolescentes embarazadas (9). Al considerarse la edad materna inferior a 20 años como factor de riesgo para óbito fetal, que suele aumentar a medida en que la madre tenga una menor edad, es posible que el bajo porcentaje del mismo en este estudio se deba al bajo número de la población objetivo (16).

Es imperativo reforzar los programas existentes para promoción de la salud sexual en la adolescencia desde el ámbito escolar y comunitario, asesoramiento individualizado en los servicios de salud y buscar estrategias para una mejor accesibilidad a los métodos anticonceptivos. Así también intervenciones educativas con énfasis en el sexo seguro promoviendo la reducción de las prácticas sexuales de riesgo y prevención de embarazo no deseado.

En conclusión, la prevalencia de embarazo adolescente en el Hospital Regional de Coronel Oviedo durante el 2011 fue del 40,7\%. Con respecto a las características epidemiológicas, se encontró mayor prevalencia de adolescentes embarazadas en etapa tardía (15 a 19 años) que provenían de la zona urbana y su estado civil era unión libre. La 
complicación más frecuente fue ruptura prematura de membrana, otras complicaciones encontradas fueron infección de las vías urinarias y óbito fetal.

La principal limitación fue la falta de información completa en las historias clínicas, por lo que en este estudio fueron estudiadas solo las variables que presentaban todas las historias clínicas.

Se recomienda realizar estudios similares anualmente para poder observar las fluctuaciones con respecto a la prevalencia de embarazo en la adolescencia, así también estudios con diseño de mayor potencia para determinar las consecuencias del embarazo adolescente en las madres y sus hijos.

\section{REFERENCIAS BIBLIOGRÁFICAS}

1. Pineda Pérez $S$, Aliño Santiago M. Manual de prácticas clínicas para la atención integral a la salud en la adolescencia. 2 ed. La Habana: Editorial MINSAP; 1999.

2. Morales Díaz E, Solanelles Rojas AM, Mora González SR, Miranda Gómez O. Embarazo no deseado en alumnas universitarias. Rev Cub Med Mil. 2013; 42 (2): 153 - 163.

3. Mancilla Ramírez J. Embarazo en adolescente. Vidas en riesgo. Perinatol. Reprod. Hum. 2012; 26 (1): 5 - 7.

4. Mora Cancino AM, Hernández Valencia M. Embarazo en la adolescencia. Ginecol Obstet Mex. 2015; 83 (5): 294 - 301.

5. Diniz Bensaja Dei Schiro E, García Dias AC, Neiva Silva L, Nieto CJ, Koller SH. Características familiares y apoyo percibido entre adolescentes brasileños con y sin experiencia del embarazo. Avances en Psicología Latinoamericana. 2012; 30 (1): 65 - 80.

6. Gómez Sotelo A, Gutiérrez Malaver ME, Izzedin Bouquet R, Sánchez Martínez LM, Herrera Medina NE, Ballesteros Cabrera M. Representaciones sociales del embarazo y la maternidad en adolescentes primigestantes y multigestantes en Bogotá. Rev. Salud pública. 2012; 14 (2): 189 - 199.

7. Organización Mundial de la Salud [Internet]. Barcelona: cepal; 2005 [citado 10 jun 2015]. Disponible en: http://www.cepal.org/mujer/ noticias/noticias/0/33810/ Embarazoadolescente.pdf

8. Osorio I, Hernández M. Prevalencia de deserción escolar en embarazadas adolescentes en instituciones educativas oficiales del Valle del Cauca, Colombia, 2006. Colomb Med. 2011; 42 (3): 303 - 308.
9. León P, Minassian M, Bordoño R, Bustamante F. Embarazo adolescente. Rev. Ped. Elec. 2008; 5 (1): 42 - 51.

10. Ulanowicz MG, Parra KE, Wendler GE, Monzón LT. Riesgos en el embarazo adolescente. Revista de posgrado de la Via Cátedra de Medicina. 2006; 153: 13 - 17.

11. Ramos, S. Intervenciones para prevenir embarazos no deseados en adolescentes: Comentario de la BSR (última revisión: 1 de agosto de 2011). La Biblioteca de Salud Reproductiva de la OMS; Ginebra: Organización Mundial de la Salud.)

12. Hernández, G: "Estrategia educativa para disminuir el embarazo en la adolescencia, en el área de salud Taguasco, 2008-2012" en Revista Caribeña de Ciencias Sociales, enero 2013, en http://caribeña.eumed.net/estrategia-educativadisminuir-embarazo-adolescencia/

13. Calero JL, Santana F. Percepciones de un grupo de adolescentes sobre iniciación sexual, embarazo y aborto. Rev Cubana Salud Pública. 2001; 27(1): 50 - 57.

14. Vázquez Márquez Á, Guerra Verdecia C, Herrera Vicente V, Chávez F, Almirall Chávez AM. Embarazo y adolescencia: Factores biológicos materno y perinatal más frecuentes. Rev Cubana Obstet Ginecol. 2001; 27(2): 158 - 164.

15. Centro Paraguayo de Estudios de Población. (CEPEP). Encuesta Nacional de Demografía y Salud Sexual y Reproductiva 2004. QR producciones gráficas. Asunción: CEPEP; 2005.

16. Padilla de Gil M. Aspectos médicos y sociales de la maternidad en la adolescencia. Rev Sogia. 2000; 7(1): 16-25.

17. Díaz A, Sanhueza P, Yaksic N. Riesgos obstétricos en el embarazo adolescente: Estudio comparativo de resultados obstétricos y perinatales con pacientes embarazadas adultas. Rev. chil. obstet. Ginecol. 2002; 67(6): 481-487.

\section{Fuente de Financiamiento}

Los autores declaran no presentar conflictos de interés.

Conflicto de interés

Los autores declaran no presentar ningún conflicto de interés.

Correspondencia:

Jorge Sebastián Escobar Salinas

Dirección: Barrio 12 de Junio. Coronel Oviedo, Paraguay.

Teléfono: 595973207996

Correo electrónico:joseessa@gmail.com

Recibido: 03 febrero de 2016 Aprobado: 30 de marzo de 2016 